\title{
Exploring the potential activity spectrum of two 5 -nitroindazolinone prototypes on different Trypanosoma cruzi strains
}

\author{
CRISTINA FONSECA-BERZAL ${ }^{1,2} *$, PATRICIA BERNARDINO DA SILVA ${ }^{3}$, \\ CRISTIANE FRANÇA DA SILVA ${ }^{3}$, MARIANE VASCONCELOS ${ }^{3}$, \\ MARCOS MEUSER BATISTA ${ }^{3}$, JOSÉ A. ESCARIO ${ }^{1,2}$, VICENTE J. ARÁN ${ }^{1,4}$, \\ ALICIA GÓMEZ-BARRIO ${ }^{1,2}$ and MARIA DE NAZARÉ C. SOEIRO ${ }^{3}$ \\ ${ }^{1}$ CEI Campus Moncloa, UCM-UPM \& CSIC, Madrid, Spain \\ ${ }^{2}$ Departamento de Parasitología, Facultad de Farmacia, Universidad Complutense de Madrid, Pza. Ramón y Cajal s/n, \\ 28040 Madrid, Spain \\ ${ }^{3}$ Laboratório de Biologia Celular, Instituto Oswaldo Cruz, Fiocruz, Av. Brasil 4365, 21040-900 Rio de Faneiro, Brazil \\ ${ }^{4}$ Instituto de Química Médica (IQM), Consejo Superior de Investigaciones Científicas (CSIC), c/Fuan de la Cierva 3 , \\ 28006 Madrid, Spain
}

(Received 3 Fuly 2015; revised 7 August 2015; accepted 24 August 2015)

\section{SUMMARY}

In the present study, the potential activity of two 5-nitroindazole derivatives previously proposed as suitable antichagasic prototypes was further evaluated on diverse Trypanosoma cruzi strains belonging to two discrete typing units (DTUs) frequently associated with human infection (i.e. DTUs TcII and TcVI). The trypanocidal profile that both 2-benzyl-1-propyl (22) and 2-benzyl-1-butyl (24) derivatives achieved on Tulahuen amastigotes $\left(\mathrm{IC}_{50}=3 \cdot 56 \pm 0.99\right.$ and $6 \cdot 31 \pm 1 \cdot 04 \mu \mathrm{M}$, respectively) correlates with that of formerly obtained on CL Brener, corroborating an outstanding activity on DTU TcVI parasites. Moreover, a sequential screening on extracellular and intracellular stages of T. cruzi Y (DTU TcII) demonstrated also the effectiveness of 22 and 24 over this strain on a similar range of activity $\left(\mathrm{IC}_{50}\right.$ epimastigotes $=3.55 \pm 0.47$ and $7 \cdot 92 \pm 1.63 \mu \mathrm{M}, \mathrm{IC}_{50}$ amastigotes $=2 \cdot 80 \pm 0 \cdot 46$ and $9 \cdot 02 \pm 5 \cdot 26 \mu \mathrm{M}$, respectively). These results, supported by a lack of toxicity registered over either L929 fibroblasts or primary cultures of cardiomyocytes, confirm that 5 -nitroindazolinones 22 and 24 display great selectivity on both drug-sensitive (CL and Tulahuen) and drug-moderately resistant (Y) T. cruzi strains, and therefore, represent an important outcome in the research of Chagas disease chemotherapy.

Key words: Chagas disease, Trypanosoma cruzi, 5-nitroindazolinone, DTU, L929 fibroblasts, cardiomyocytes.

\section{INTRODUCTION}

Chagas disease (CD), caused by the kinetoplastid haemoflagellate Trypanosoma cruzi, is a parasitic infection naturally transmitted in 21 Latin American countries by the contaminated feces of bloodsucking bugs (i.e. triatomines). In the endemic area, CD provokes more than 7000 deaths per year and maintains over 25 million people at risk for the infection (WHO, 2015). Moreover, its emerging character in non-endemic areas due to population mobility and alternative routes of transmission (e.g. blood transfusion, organ donation, mother-tochild and through contaminated food) (Schmunis and Yadon, 2010) has contributed to the spreading of an illness that currently affects about 7 million people worldwide (WHO, 2015).

As one of the 17 tropical diseases defined by the WHO as neglected (WHO, 2015), strategies of research and development are focused on the finding

* Corresponding author: Departamento de Parasitología, Facultad de Farmacia, Universidad Complutense de Madrid, Pza. Ramón y Cajal s/n, Madrid 28040, Spain. E-mail: crfonseca@pdi.ucm.es of a suitable CD chemotherapy (Zingales et al. 2014). Many efforts have been made by institutions in order to develop new compounds potentially applicable to the treatment of $\mathrm{CD}$, since the available chemotherapy relies on two old nitroheterocyclic drugs: benznidazole (BZ), the first-line treatment in most countries, and nifurtimox (NX) (WHO, 2015). Although these medicines are currently accepted to treat the acute and the earlychronic disease, both show limited effectiveness in long-term chronic infections (Urbina, 2015) and also exhibit undesirable side effects (Castro et al. 2006).

Concerning experimental chemotherapy research, in a previous study we proposed the 1,2-disubstituted 5-nitroindazolinone scaffold as prototype of antichagasic drug (Vega et al. 2012). Concretely, the 2-benzyl-1-propyl (22) and the 2-benzyl-1butyl (24) derivatives (Fig. 1) achieved outstanding activity over the replicative stages of $T$. cruzi (i.e. epimastigotes and amastigotes) without toxicity on macrophages, what led to great selectivity on CL Brener strain (Vega et al. 2012; Fonseca-Berzal et al. 2014).

Parasitology Open (2015), Vol. 1, e1; page 1 of 10. (C) Cambridge University Press 2015. This is an Open Access article, distributed under the terms of the Creative Commons Attribution licence (http://creativecommons.org/licenses/by/4.0/), which permits unrestricted re-use, distribution, and reproduction in any medium, provided the original work is properly cited. 


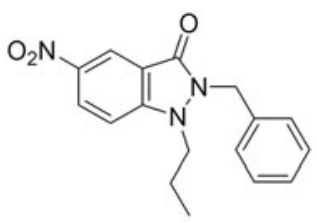

22

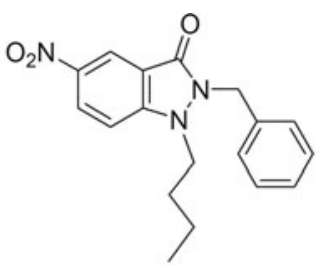

24
Fig. 1. Chemical structures of the prototypes 2-benzyl-5nitro-1-propylindazolin-3-one (22) and 2-benzyl-1-butyl5-nitroindazolin-3-one (24).

Trypanosoma cruzi populations have been classified into six discrete typing units (DTUs TcI-TcVI) according to molecular genetics, eco-epidemiological features and pathogenicity (Zingales et al. 2014). Moreover, susceptibility to reference drugs also varies among $T$. cruzi strains, with CL Brener and Tulahuen ( $\mathrm{TcVI}$ ) known as drug-sensitive, $\mathrm{Y}$ (TcII) as drug-moderately resistant and Colombian (TcI) as drug-resistant strain (Soeiro et al. 2013).

In this context, the Parasitology Department of UCM (Madrid, Spain), the Medicinal Chemistry Institute of CSIC (Madrid, Spain) and the Cellular Biology Laboratory of IOC/Fiocruz (Rio de Janeiro, Brazil) have worked in collaboration to further explore the trypanocidal spectrum of these candidates, following routinely screening procedures standardized by these laboratories. Consequently, the aims of the present study were: (i) to analyse the trypanocidal profile of prototypes 22 and 24 on other $T$. cruzi strains belonging to DTUs involved in human infection; (ii) to evaluate their activity in an in vitro golden model that uses primary cultures of cardiac cells as mammalian hosts since heart is one of the main targets for CD infection and inflammation; and (iii) to confirm their lack of toxicity over different mammalian cell cultures.

MATERIALS AND METHODS

\section{Ethics}

All procedures involving mice were carried out in accordance with the guidelines established by the Fiocruz Committee of Ethics for the Use of Animals (CEUA LW16/14).

\section{Compounds}

The synthesis of the two 5-nitroindazole derivatives assayed in the present work (Fig. 1) was previously described (Vega et al. 2012). The numbering of compounds used in this reference has been followed in the present paper. For all the in vitro assays, stock solutions of 22 and 24 were prepared in dimethyl sulfoxide and extemporaneously added to the cultures in a final concentration of the solvent non-toxic itself $(<1 \%, v / v)$. BZ (Laboratório
Farmacêutico do Estado de Pernambuco - LAFEPE, Brazil) was assayed as a reference drug.

\section{Mammalian cell cultures}

Primary cultures of embryonic cardiomyocytes (CM) were obtained from Swiss mice as previously described (Meirelles et al. 1986). After their purification, CM cultures were sustained in Dulbecco's Modified Eagle's Medium (DMEM) supplemented with $2.5 \mathrm{mM} \mathrm{CaCl}_{2}, 1 \mathrm{mM}$ L-glutamine, $5 \%$ heatinactivated fetal bovine serum (FBS) (30 min, $56{ }^{\circ} \mathrm{C}$ ) and $2 \%$ chicken embryo extract. CM cultures were maintained at $37^{\circ} \mathrm{C}$ in a humidified atmosphere of $5 \% \mathrm{CO}_{2}$.

Murine L929 fibroblasts were grown in plastic culture flasks $\left(75 \mathrm{~cm}^{2}\right)$ using either Minimal Essential Medium (MEM) without phenol-red and supplemented as reported (Fonseca-Berzal et al. 2014) (MEMS) or RPMI-1640 without phenol-red supplemented with $10 \%$ heat-inactivated FBS and $2 \mathrm{~mm}$ glutamine (RPMIS). L929 cultures were maintained in a humidified $5 \% \mathrm{CO}_{2}$ atmosphere at $37^{\circ} \mathrm{C}$ and subpassaged once a week. A $0 \cdot 03 \%$ ethylenediaminetetraacetic acid (ED'TA) and $0.05 \%$ trypsin in phosphate-buffered saline (PBS) solution was used for cell detachment.

\section{Parasites}

The Y strain of T. cruzi, originally isolated from an acute human case (Silva and Nussenzweig, 1953) and the Tulahuen strain, stably transfected with the Escherichia coli $\beta$-galactosidase gene lac $Z$ (Buckner et al. 1996) were used throughout the experiments.

Regarding the Y strain, epimastigotes were maintained at $28^{\circ} \mathrm{C}$ in supplemented liver infusion tryptose (LIT) medium as described in (Vega et al. 2012) and the axenic cultures continuously maintained in logarithmic growth by weekly passages. Bloodstream trypomastigotes (BT) of this strain were obtained by heart puncture from infected Swiss mice at the parasitaemia peak day and after their purification, resuspended in RPMI medium supplemented with 5\% heat-inactivated FBS.

Concerning Tulahuen parasites, tissue culturederived trypomastigotes (TCT) of this $\beta$-galactosidase-transfected strain were harvested in the supernatant of L929 cultures previously infected with invasive forms of $T$. cruzi and maintained in RPMIS at $37{ }^{\circ} \mathrm{C}$ in a humidified $5 \% \mathrm{CO}_{2}$ atmosphere (Romanha et al. 2010).

\section{Epimastigote susceptibility assay}

The activity on epimastigotes was evaluated by applying the resazurin assay previously standardized by Rolón et al. (2006). Log-phase epimastigotes in LIT medium were seeded in the culture tubes at 
a density of $3 \times 10^{6}$ parasites $\mathrm{mL}^{-1}$ and maintained at $28{ }^{\circ} \mathrm{C}$ overnight to allow homogeneous growth. Afterwards, cultures were distributed in 96-well microplates $(200 \mu \mathrm{L}$ perwell) and incubated within the compounds for $48 \mathrm{~h}$ at $28^{\circ} \mathrm{C}$. Growth, medium and drug controls were also included in each assay and concentrations tested in triplicate. Finally, $20 \mu \mathrm{L}$ of a resazurin solution in $1 \%$ PBS ( $3 \mathrm{~mm}, \mathrm{pH}$ 7 ) was added per well and the plates maintained at $28^{\circ} \mathrm{C}$ for another $5 \mathrm{~h}$. Fluorescence intensity was measured with excitation $(535 \mathrm{~nm})$ and emission $(590 \mathrm{~nm})$ wavelengths (alamarBlue ${ }^{\circledR}$ Assay, U.S. Patent No. 5501 959) and the results represent the percentage of epimastigote growth inhibition (\% EGI). For each compound, the concentration that inhibits $50 \%$ of epimastigote growth $\left(\mathrm{IC}_{50}\right)$ was estimated by plotting the concentrations tested $v$ s the \% EGI. Selectivity indexes (SI) and potencies relative to BZ (RP) were also estimated.

\section{Cytotoxicity assays}

In order to detect any potential toxicity towards the host cell, cultures of CM and L929 were incubated within these compounds and the metabolic cell function was measured in the presence of resazurin-based indicators (PrestoBlue ${ }^{\circledR}$ and Resazurin sodium salt, respectively).

According to this, $100 \mu \mathrm{L}$ of DMEM containing $6 \times 10^{4} \mathrm{CM}$ per well were seeded in 96 -well microplates previously coated with gelatin and incubated overnight at $37{ }^{\circ} \mathrm{C}$ in a humidified $5 \% \mathrm{CO}_{2}$ atmosphere. Afterwards, the medium was replaced by solutions of each compound in fresh DMEM and the plates were incubated either 24 or $48 \mathrm{~h}$ in the conditions aforementioned. Each concentration was evaluated in triplicate and controls of cellular growth were included in all the plates. Once the incubation concluded, both cell morphology and contractibility were examined by light microscopy and cellular viability evaluated by adding the redox indicator PrestoBlue ${ }^{\circledR}$ according to the manufacturer's instructions. After $5 \mathrm{~h}$ of incubation at $37^{\circ} \mathrm{C}$ in a humidified $5 \% \quad \mathrm{CO}_{2}$ atmosphere, the absorbance was read at 570 and $600 \mathrm{~nm}$ and the results were expressed as the percentage of cytotoxicity on CM $\left(\% \mathrm{C}_{\mathrm{CM}}\right)$ (Romanha et al. 2010).

Moreover, the unspecific cytotoxicity over L929 was assayed in 96 -well plates by seeding either $10 \times 10^{3}$ or $15 \times 10^{3}$ cells in $100 \mu \mathrm{L}$ of MEM per well. In order to allow cell attachment, the plates were incubated for $3 \mathrm{~h}$ at $37^{\circ} \mathrm{C}$ in a humidified $5 \%$ $\mathrm{CO}_{2}$ atmosphere and then, the medium was replaced by $200 \mu \mathrm{L}$ of compounds diluted in fresh MEM. Each concentration was tested in triplicate. Growth, medium and drug controls were also included in each plate. Fibroblasts were exposed to the compounds for 48,72 and $96 \mathrm{~h}$ at $37^{\circ} \mathrm{C}$ with $5 \% \mathrm{CO}_{2}$. Afterwards, $20 \mu \mathrm{L}$ of a resazurin in $1 \%$ PBS solution
( $2 \mathrm{mM}, \mathrm{pH} 7$ ) was added to each well and the plates were returned to the incubator for another $3 \mathrm{~h}$. Finally, fluorescence intensity was read at $535 \mathrm{~nm}$ (excitation) and $590 \mathrm{~nm}$ (emission) and the results were expressed as \% $\mathrm{C}_{\mathrm{L} 929}$ (Fonseca-Berzal et al. 2015).

For both assays, the concentration that inhibits $50 \%$ of cellular growth $\left(\mathrm{LC}_{50}\right)$ was estimated by plotting drug concentrations vs $\% \mathrm{C}$.

\section{Intracellular amastigote susceptibility assays}

The activity on intracellular amastigotes was evaluated by infecting either L929 fibroblasts with TCT (Tulahuen strain) or CM with $\mathrm{BT}$ (Y strain) in a 10:1 ratio (parasite:cell).

For the first bioassay, $100 \mu \mathrm{L}$ of RPMIS containing 4000 L929 cells per well was seeded in 96-well tissue culture plates and maintained for $24 \mathrm{~h}$ at $37^{\circ} \mathrm{C}$ in a humidified 5\% $\mathrm{CO}_{2}$ atmosphere. Afterwards, cells were incubated within TCT for another $2 \mathrm{~h}$ and then, non-penetrated parasites were discarded replacing the culture medium by fresh RPMIS. In order to establish the infection, the plates were maintained during $48 \mathrm{~h}$ at $37^{\circ} \mathrm{C}$ and $5 \% \mathrm{CO}_{2}$ and then, the medium was replaced by solutions of each compound in fresh RPMIS. Each concentration was evaluated in triplicate. Controls of infection and cell growth were also included and the plates were incubated for $96 \mathrm{~h}$ at the same conditions of temperature and humidity. After this period, $50 \mu \mathrm{L}$ of $500 \mu \mathrm{M}$ chlorophenol red glycoside in $0.5 \%$ Nonidet $\mathrm{P} 40$ was added to each well and the plates were incubated for $18 \mathrm{~h}$ at $37^{\circ} \mathrm{C}$. Finally, the absorbance was read at $570 \mathrm{~nm}$ and the results were expressed as the percentage of amastigote growth inhibition (\%AGI) (Romanha et al. 2010).

To assay these compounds over Y strain amastigotes, $100000 \mathrm{CM}$ per well were seeded in 24-well tissue culture plates provided with round coverslips previously coated with gelatin and then, maintained in DMEM overnight at $37^{\circ} \mathrm{C}$ in a humidified $5 \%$ $\mathrm{CO}_{2}$ atmosphere. After $24 \mathrm{~h}$ of parasite-host cell interaction, the infected cultures were washed to remove non-internalized trypomastigotes and then, incubated for another $48 \mathrm{~h}$ within the compounds diluted in fresh DMEM. Next, the cultures were fixed with Bouin's fixative and stained with Giemsa. The mean number of infected CM and the mean number of parasites per infected CM were scored in 400 host cells by duplicate. Only parasites with characteristic nuclei and kinetoplast were counted, since the irregular ones were considered as parasites undergoing death. Finally, activity results were estimated by calculating the inhibition of the endocytic index (EI) (da Silva et al. 2007).

In each assay, compounds $\mathrm{IC}_{50}$ and $\mathrm{IC}_{90}$ (concentration that inhibits 50 and $90 \%$ of amastigote proliferation or EI, respectively) were estimated by plotting concentrations vs \%AGI or \%EI. SI over the respective host cell and RP were also calculated. 

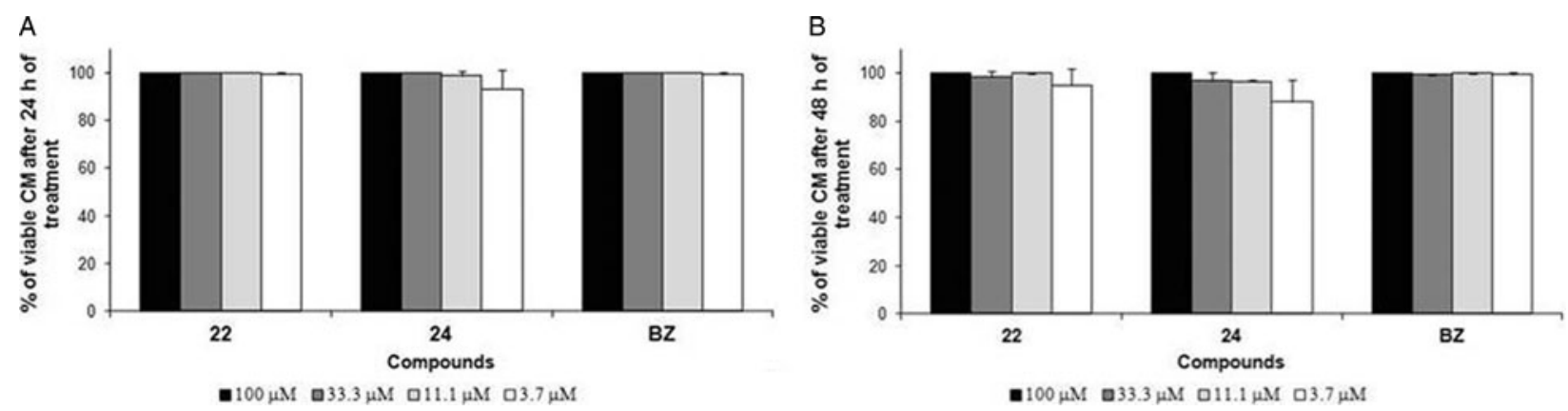

Fig. 2. Toxic effect of the 5-nitroindazolinones over primary cultures of CM after $24 \mathrm{~h}(\mathrm{~A})$ and $48 \mathrm{~h}(\mathrm{~B})$ of treatment.

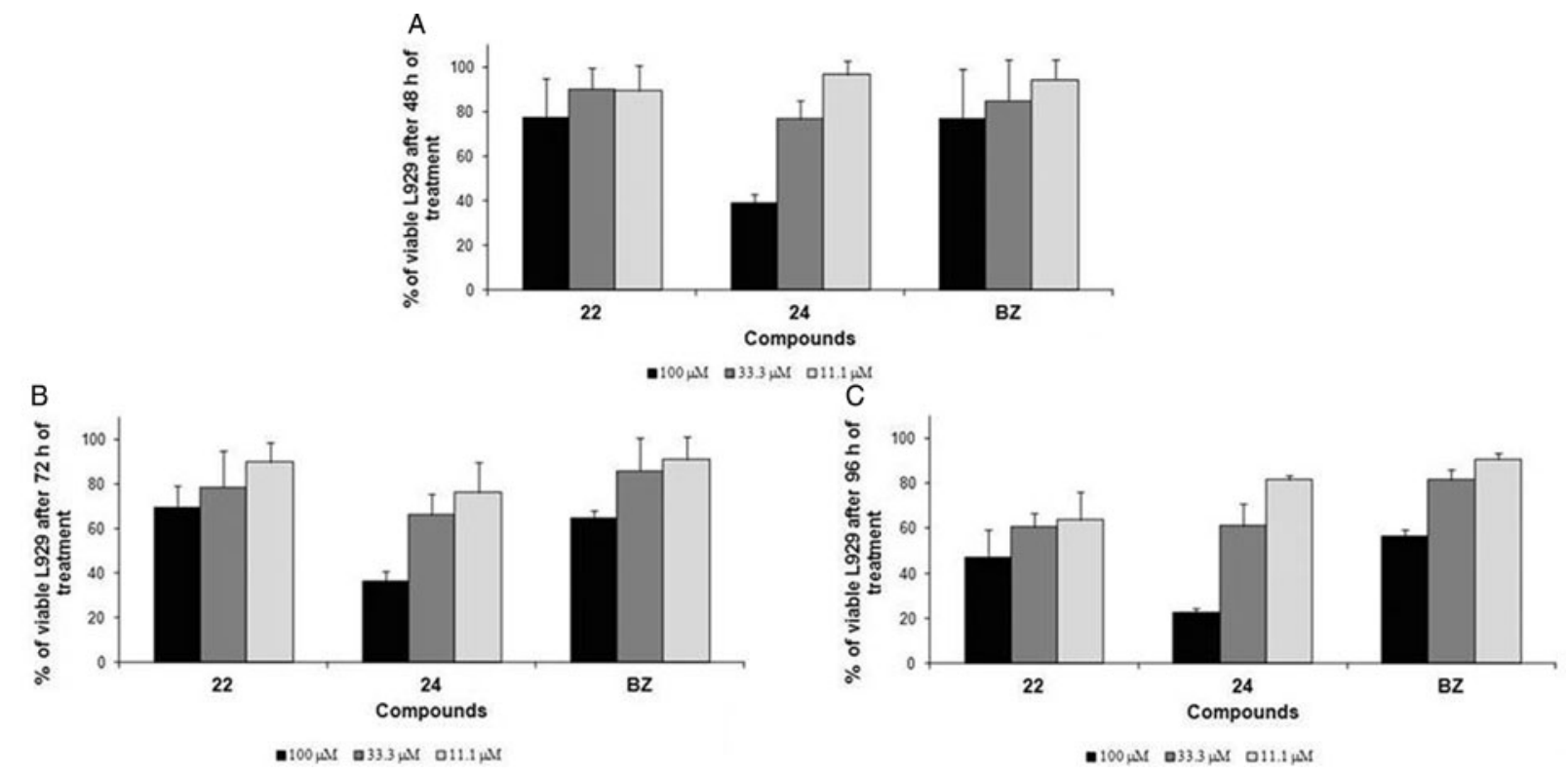

Fig. 3. Toxic effect of the 5-nitroindazolinones over L929 cells after $48 \mathrm{~h}$ (A), $72 \mathrm{~h} \mathrm{(B)}$ and $96 \mathrm{~h}$ (C) of treatment.

All the in vitro assays were run in the same conditions at least twice separately. For each assay, the results are expressed as the mean value of activity \pm standard deviation (S.D.).

\section{Statistical analysis}

SPSS Statistics software (version 20, IBM) was used for the statistical analysis. The non-parametric Kruskal-Wallis test was applied to compare compounds activity between the different strains, as well as with that of BZ. $P<0.05$ was considered statistically significant.

\section{RESULTS}

\section{Toxicity on mammalian host cells}

Since both CM and L929 were used as T. cruzi host cell, the potential toxicity of 22 and 24 over these mammalian cells was assessed in vitro. As Fig. 2 reflects, no toxicity was perceived on the treated CM, and therefore, percentages of cell viability greater than $85 \%$ were registered in all the cases. In fact, both derivatives achieved $\mathrm{LC}_{50}$ values higher than $100 \mu \mathrm{M}$ after the incubation times assayed (i.e. 24 and $48 \mathrm{~h}$ ). Moreover, the light microscopy analysis revealed neither alteration in cell morphology nor affectation in contractibility as a treatment consequence.

Nevertheless, both compounds achieved a different cytotoxic profile on L929 fibroblasts. After $48 \mathrm{~h}$ of drug-cell contact, concentrations higher than 33.3 $\mu \mathrm{v}$ led to a pronounced loss of cellular viability that varied in a time-dependent manner. Likewise, BZ induced higher toxicity on this mammalian cell line, showing a response similar to that of derivative 22 (Fig. 3 and Table 1). However, at the highest concentration tested and after completing the three incubation times assayed on L929, no differences were detected between the toxic profile of 5-nitroindazolinones and BZ $(P>0 \cdot 05)$.

\section{Activity on epimastigotes ( $Y$ strain)}

As a primary screening, the inhibitory effect of derivatives 22 and 24 was evaluated on axenic cultures of epimastigotes. Both compounds displayed a better trypanocidal profile compared with that of 
Table 1. Toxic effect of derivatives 22 and 24 on L929 cells

\begin{tabular}{|c|c|c|c|c|c|c|}
\hline \multirow[b]{2}{*}{ Compound } & \multicolumn{2}{|l|}{$48 \mathrm{~h}$} & \multicolumn{2}{|l|}{$72 \mathrm{~h}$} & \multicolumn{2}{|l|}{$96 \mathrm{~h}$} \\
\hline & $\% \mathrm{C}^{\mathrm{a}}$ & $\mathrm{LC}_{50}(\mu \mathrm{M})^{\mathrm{b}}$ & $\% \mathrm{C}$ & $\mathrm{LC}_{50}(\mu \mathrm{M})$ & $\% \mathrm{C}$ & $\mathrm{LC}_{50}(\mu \mathrm{M})$ \\
\hline 22 & $22 \cdot 74 \pm 17 \cdot 74$ & $>100$ & $30 \cdot 70 \pm 9 \cdot 98$ & $>100$ & $52 \cdot 66 \pm 12 \cdot 34$ & $86 \cdot 57 \pm 10 \cdot 77$ \\
\hline 24 & $60 \cdot 97 \pm 3 \cdot 94$ & $79 \cdot 79 \pm 4 \cdot 64$ & $63 \cdot 59 \pm 4 \cdot 26$ & $67 \cdot 44 \pm 5 \cdot 48$ & $77 \cdot 39 \pm 1 \cdot 75$ & $55 \cdot 34 \pm 7 \cdot 89$ \\
\hline $\mathrm{BZ}$ & $35 \cdot 13 \pm 14 \cdot 33$ & $>100$ & $35 \cdot 17 \pm 3 \cdot 27$ & $>100$ & $43 \cdot 52 \pm 2 \cdot 79$ & $>100$ \\
\hline
\end{tabular}

* Significant differences $(P<0 \cdot 05)$ compared with BZ (Kruskal-Wallis test).

a Results expressed as the mean value of cytotoxicity (\%C) at the highest concentration tested $(100 \mu \mathrm{M}) \pm$ standard deviation (S.D.) of three independent experiments $(n=3)$.

b The concentration causing $50 \%$ of cellular lethality $\left(\mathrm{LC}_{50}\right)$ was estimated by plotting drug concentrations $v s \% \mathrm{C}$. The results are expressed as the mean \pm S.D. of three independent experiments $(n=3)$.
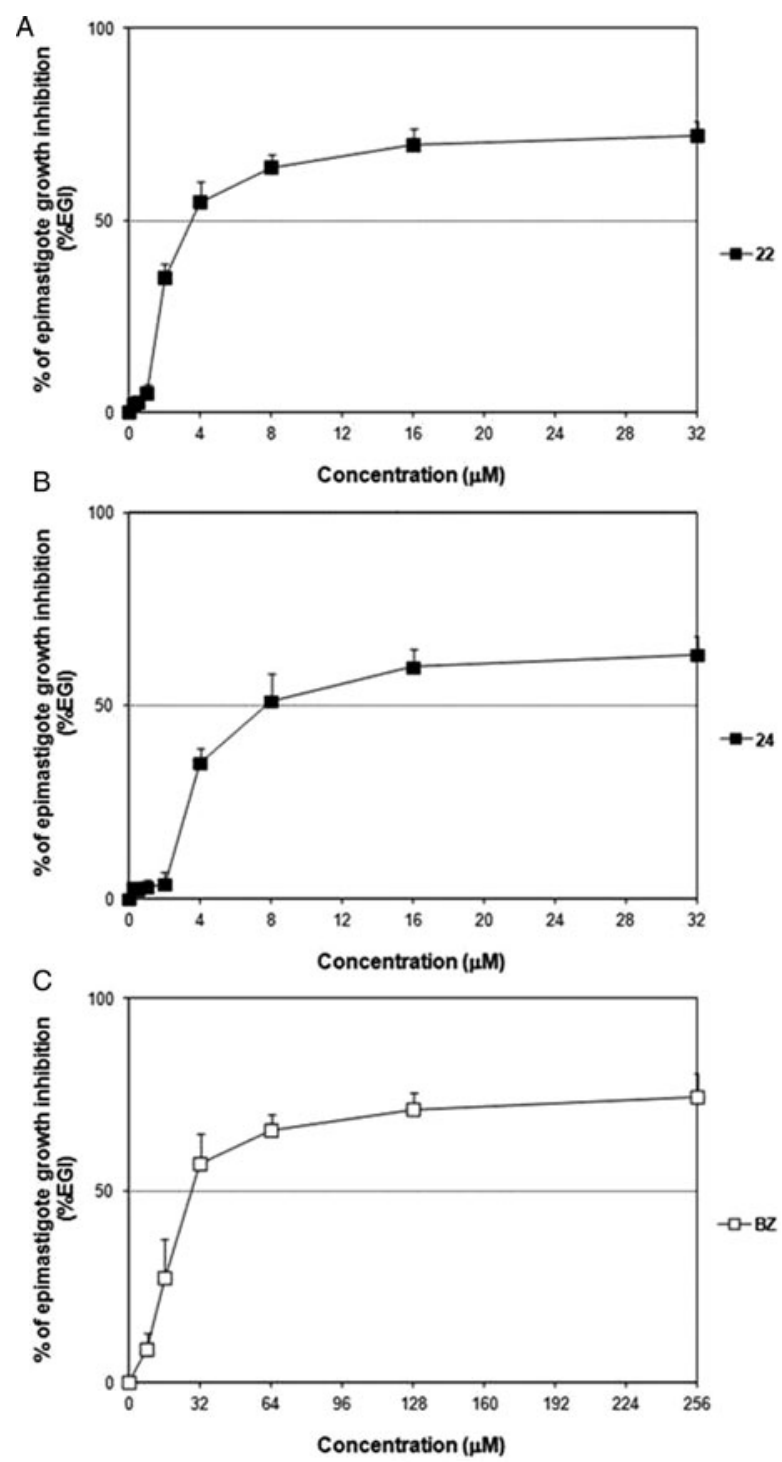

Fig. 4. Dose-response curves of 22 (A), 24 (B) and BZ (C) on epimastigotes (Y strain).

BZ (Fig. 4). They obtained improved $\mathrm{IC}_{50}$ values and remarkable SI on the extracellular parasite (Table 2), significantly better in the case of derivative $22(P<$ $0 \cdot 05)$. Otherwise, the potency of these molecules could not be determined at this level, since their
$\mathrm{IC}_{90}$ were reached at concentrations superior than the highest one assayed.

\section{Activity on intracellular amastigotes}

The inhibitory effect of 22 and 24 was further evaluated over Tulahuen and $\mathrm{Y}$ strain amastigotes grown in L929 and CM cultures, respectively. According to the results of cytotoxicity (Table 1), concentrations higher than $50 \mu \mathrm{M}$ were not tested at this level. Although both compounds displayed slightly lower dose-response curves upon Tulahuen parasites compared with that of BZ (Fig. 5 and Table 3), they reached outstanding SI on the intracellular stage and were as potent $(P>0.05)$ as the reference drug ('Table 3).

Regarding the activity upon Y strain amastigotes, although compound 22 achieved similar $\mathrm{IC}_{50}$ values for both the strains $(P>0 \cdot 05)$, it accomplished a better $\mathrm{IC}_{90}$ after a 2-day treatment of $\mathrm{Y}$-infected cells and a nearly suppression of the EI at $25 \mu \mathrm{M}$ $(\% \mathrm{EI}=99 \cdot 43 \pm 0 \cdot 32 \%)($ Fig. 6$)$, showing a trypanocidal profile similar to that of BZ (Table 4). Otherwise, derivative 24 was not only less effective but also with lower potency on this latter strain (Table 4). However, at $25 \mu \mathrm{M}$ it obtained a considerable reduction in both the percentage of infected cells and the mean number of amastigotes per infected cell, as the inhibition in the EI reflects (\%EI ca. 71\%) (Figs 6 and 7).

\section{DISCUSSION}

The selection of diverse T. cruzi strains and clones for in vitro drug screening is an important point to consider in the early research of putative candidates for CD chemotherapy (Zingales et al. 2014). In fact, the different effectiveness that BZ and NX display among divergent strains is one of the aspects on which the current Chagas therapy failure resides (Urbina, 2015). Although a clear correlation between T. cruzi genetic variability and its presumable response to drugs has not been evidenced (Moraes et al. 2014), strategies for drug screening 
Table 2. Trypanocidal activity of 22 and 24 on $\mathrm{Y}$ strain epimastigotes expressed as $\mathrm{IC}_{50}$, IC 90 and $\mathrm{SI}$

\begin{tabular}{lllll}
\hline \hline Compound & $\mathrm{IC}_{50}(\mu \mathrm{M})^{\mathrm{a}}$ & $\mathrm{IC}_{90}(\mu \mathrm{M})$ & $\mathrm{LC}_{50} \mathrm{CM}(\mu \mathrm{M})^{\mathrm{b}}$ & $\mathrm{SI}^{\mathrm{c}}$ \\
\hline 22 & $3 \cdot 55 \pm 0 \cdot 47^{* * * *}$ & $>32$ & $>100$ & $>28 \cdot 17$ \\
24 & $7 \cdot 92 \pm 1 \cdot 63^{* *}$ & $>32$ & $>100$ & $>12 \cdot 62$ \\
$\mathrm{BZ}$ & $28 \cdot 00 \pm 4 \cdot 64$ & $>256$ & $>100$ & $>3 \cdot 57$ \\
\hline \hline
\end{tabular}

* Significant differences $(P<0 \cdot 05)$ compared with BZ (Kruskal-Wallis test).

* Significant differences $(P<0 \cdot 05)$ compared with the activity on CL-B5 epimastigotes (see Vega et al. 2012) (KruskalWallis test).

a The concentration causing 50 and $90 \%$ of EGI ( $\mathrm{IC}_{50}$ and $\mathrm{IC}_{90}$, respectively) was estimated by plotting drug concentrations vs \%EGI. The results are expressed as the mean \pm S.D. of three independent experiments $(n=3)$.

b After $48 \mathrm{~h}$ of drug treatment.

c Selectivity indexes are defined as $\mathrm{SI}=\mathrm{LC}_{50}(48 \mathrm{~h} \mathrm{CM}$ cells $) / \mathrm{IC}_{50} \mathrm{Y}$ epimastigotes.
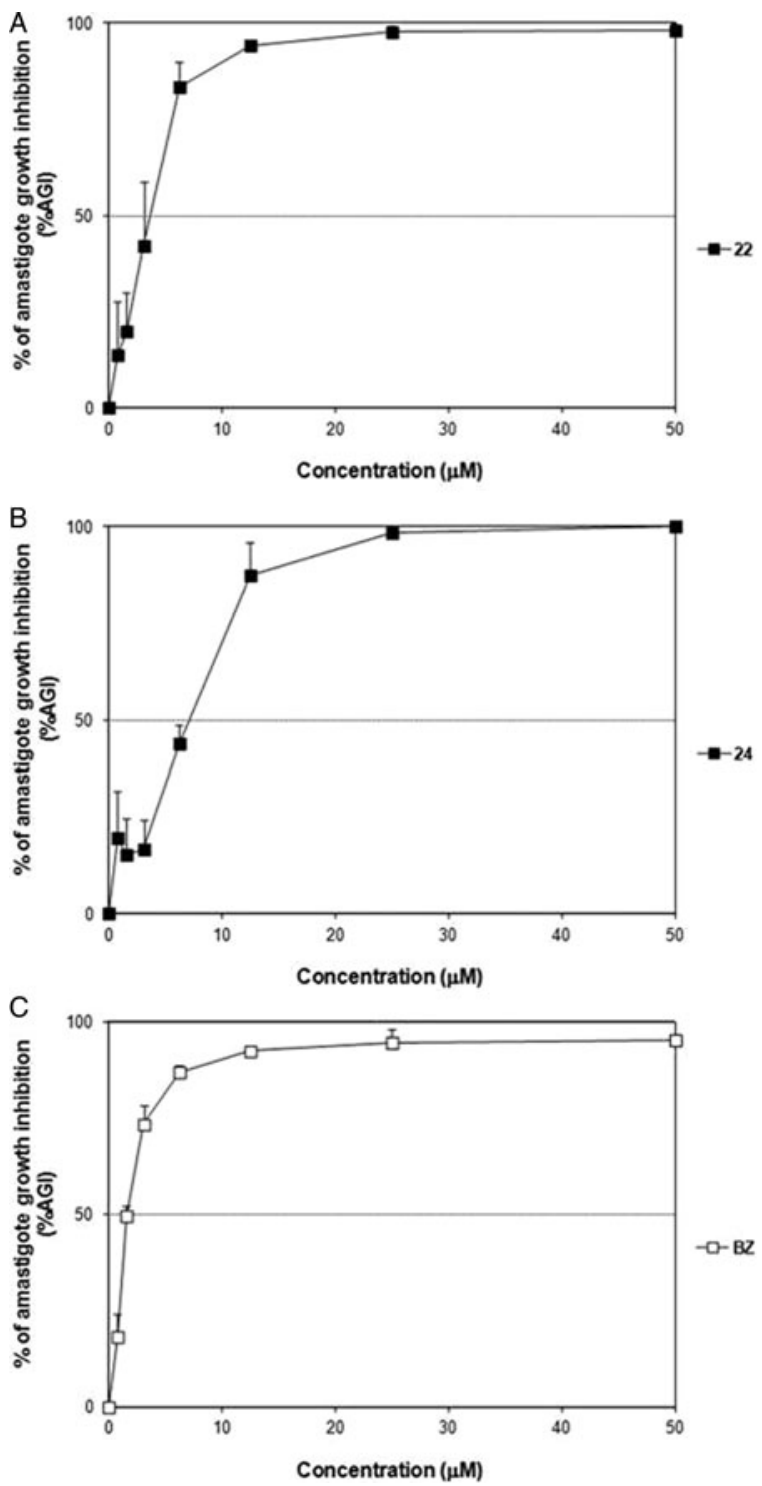

Fig. 5. Dose-response curves of 22 (A), 24 (B) and BZ (C) on intracellular amastigotes (Tulahuen strain) grown in L929 cultures

should focus its performance on parasite DTUs more often associated with human infection (DTUs TcI, TcII, TcV and TcVI) (Zingales et al. 2014).
In previous studies, indazole-based compounds have demonstrated great effectiveness on different T. cruzi strains (reviewed in Aguilera-Venegas et al. 2013). Concretely, several series of 5-nitroindazole derivatives have achieved important outcomes exhibiting notable anti-T. cruzi activity (Arán et al. 2005; Boiani et al. 2009; Vega et al. 2012; Muro et al. 2014), being some of these molecules proposed to trigger reactive oxygen species (ROS)-based mechanisms of action (Folch-Cano et al. 2010).

In this framework, we have formerly identified the 5 -nitroindazolinones 22 and 24 as promising antichagasic candidates, since the great activity they achieved on epimastigotes $\left(\mathrm{IC}_{50}=0.93\right.$ and 1.17 $\mu \mathrm{M}$, respectively) and intracellular amastigotes $\left(\mathrm{IC}_{50}<1\right.$ and $3 \cdot 71 \mu \mathrm{M}$, respectively) of $T$. cruzi $\mathrm{CL}$ Brener (Vega et al. 2012; Fonseca-Berzal et al. 2014). The results of this primary screening on a $\beta$-galactosidase-transfected clone (Buckner et al. 1996) with a biological behaviour similar to that of the parental CL strain (TcVI) (Le-Senne et al. 2002), prompted us to perform a deeper study of their broad activity on other T. cruzi strains (Zingales et al. 2014). Consequently, further screening procedures were carried out on intracellular forms of Tulahuen strain to confirm their activity on TcVI parasites, as well as over both extracellular and intracellular stages of $T$. cruzi Y, representative strain of TcII.

Moreover, an analysis of compounds relative cytotoxicity was simultaneously conducted. Although both candidates did not exert any toxic effect on J774 macrophages after $24 \mathrm{~h}$ of drug exposure (Vega et al. 2012), it is important to verify this fact over those mammalian cells hosting the parasite in subsequent trypanocidal assays (Fonseca-Berzal et al. 2014). In addition, either CM or fibroblasts are preferred as host cell for such an experimental model, since both are $T$. cruzi target cells more strictly involved in the pathology of the disease and only some parasite isolates concentrate infection in the mononuclear phagocytic system (Teixeira et al. 2006). Moreover, heart is an important target for infection and inflammation in CD pathology and 
Table 3. Trypanocidal activity of 22 and 24 on intracellular amastigotes of Tulahuen strain expressed as $\mathrm{IC}_{50}$, $\mathrm{IC}_{90}$ and SI. Compounds RP were also estimated

\begin{tabular}{llllll}
\hline \hline Compound & $\mathrm{IC}_{50}(\mu \mathrm{M})^{\mathrm{a}}$ & $\mathrm{IC}_{90}(\mu \mathrm{M})$ & $\mathrm{LC}_{50} \mathrm{~L} 929(\mu \mathrm{M})^{\mathrm{b}}$ & $\mathrm{SI}^{\mathrm{c}}$ & $\mathrm{RP}^{\mathrm{d}}$ \\
\hline 22 & $3 \cdot 56 \pm 0 \cdot 99$ & $9 \cdot 60 \pm 1 \cdot 17$ & $86 \cdot 57 \pm 10 \cdot 77$ & $24 \cdot 32$ & $1 \cdot 00$ \\
24 & $6 \cdot 31 \pm 1 \cdot 04 *$ & $15 \cdot 55 \pm 4 \cdot 86$ & $55 \cdot 34 \pm 7 \cdot 89$ & $8 \cdot 77$ & $0 \cdot 62$ \\
$\mathrm{BZ}$ & $1 \cdot 66 \pm 0 \cdot 10$ & $9 \cdot 63 \pm 0 \cdot 37$ & $>100$ & $>60 \cdot 24$ & - \\
\hline \hline
\end{tabular}

* Significant differences $(P<0 \cdot 05)$ compared with BZ (Kruskal-Wallis test).

** Significant differences $(P<0 \cdot 05)$ compared with the activity on CL-B5 amastigotes (see Fonseca-Berzal et al. 2014) (Kruskal-Wallis test).

a The concentration causing 50 and $90 \%$ of amastigote growth inhibition $\left(\mathrm{IC}_{50}\right.$ and $\mathrm{IC}_{90}$, respectively) was estimated by plotting drug concentrations $v$ \%AGI. The results are expressed as the mean \pm s.D. of three independent experiments $(n=3)$.

b After $96 \mathrm{~h}$ of drug treatment (see Table 1).

c Selectivity indexes are defined as $\mathrm{SI}=\mathrm{LC}_{50}(96 \mathrm{~h} \mathrm{L929}$ cells $) / \mathrm{IC}_{50}$ Tulahuen amastigotes.

$\mathrm{d}$ Relative potencies are defined as $\mathrm{RP}=\mathrm{IC}_{90} \mathrm{BZ} / \mathrm{IC}_{90}$ tested compound.
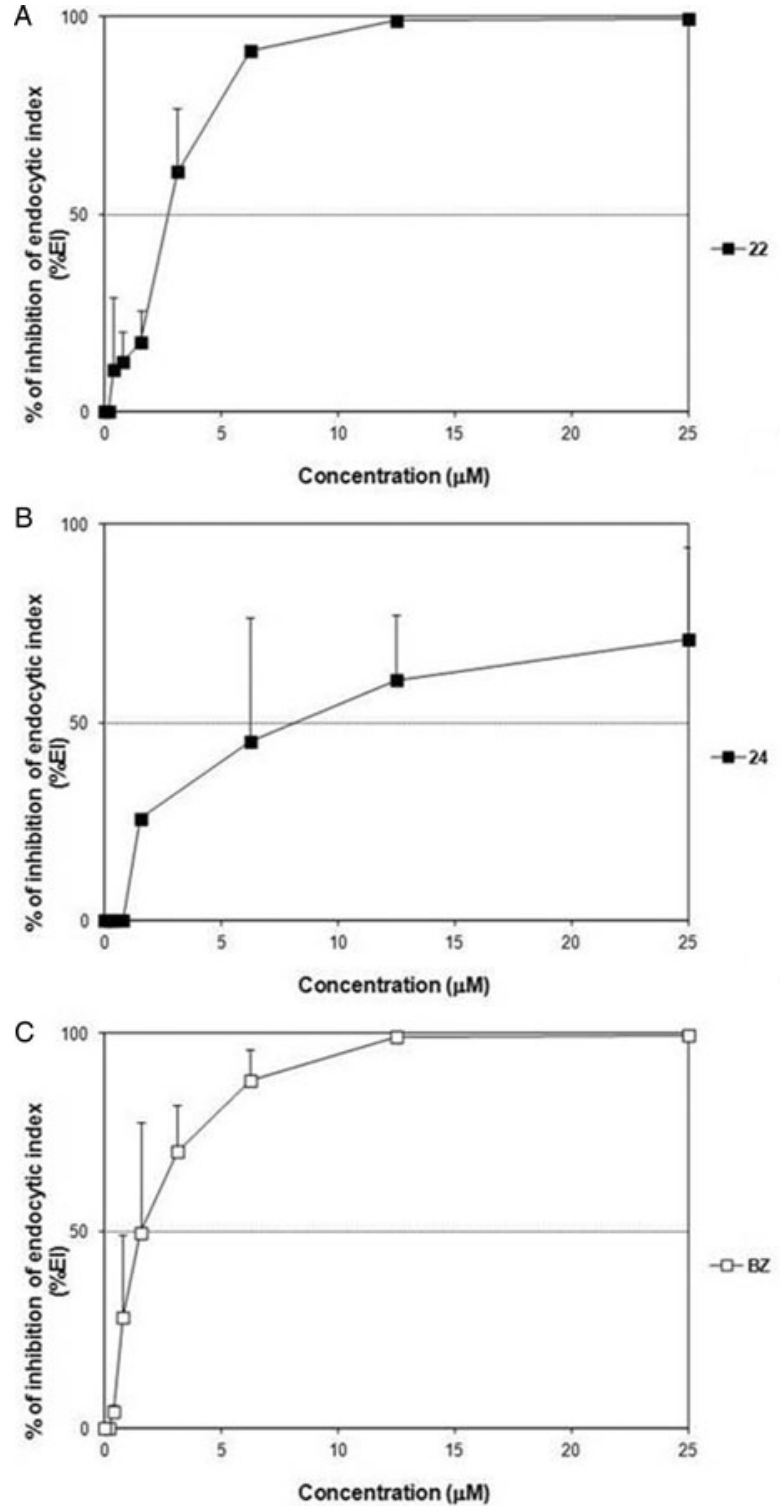

Fig. 6. Dose-response curves of 22 (A), 24 (B) and BZ (C) on intracellular amastigotes (Y strain) grown in $\mathrm{CM}$ cultures.

then, pre-clinical studies using these cells are desirable to exclude potential drug candidates with cardiotoxicity characteristics (da Silva et al. 2007).
According to this, we tested compounds 22 and 24 over uninfected L929 fibroblasts and primary cultures of CM in order to rule out the toxic effects. Neither compound 22 nor 24 resulted in toxicity on CM after completing $48 \mathrm{~h}$ of incubation (Fig. 2). Otherwise, high concentrations of both derivatives seemed to induce a loss of L929 viability that increased in a time-dependent manner, showing a behaviour similar to that of $\mathrm{BZ}(P>0 \cdot 05)$ (Fig. 3 and Table 1). It could happen as a result of the different nature of both mammalian cell cultures (i.e. primary cultures and continuous cell line, respectively) (Duran-Rehbein et al. 2014), having obtained a similar pattern with the reference drug (Figs 2 and 3 ). In fact, differences related to susceptibility and sensitivity using the same drug have been also reported according to the mammalian cell type and the respective viability assay employed ( $Z$ wolak, 2014; Emter and Natsch, 2015; Xu et al. 2015). Herein, the noticed differences in drug sensitivity detected among fibroblasts and cardiac cells may arise due to the different cytotoxic assays performed (Resazurin sodium salt $\times$ PrestoBlue ${ }^{\circledR}$ ) and from the dissimilar impact susceptibility of each target cell (fibroblasts $v s$ cardiac cells).

Several studies have reported no apparent DTU association regarding epimastigote susceptibility to both reference drugs (Boiani et al. 2006; Moreno et al. 2010; Zingales et al. 2014) agreeing this statement with the results we achieved with $\mathrm{BZ}$ on CL-B5 $\left(\mathrm{IC}_{50}=27 \cdot 32 \mu \mathrm{M}\right)($ Vega et al. 2012) and $\mathrm{Y}$ strain $\left(\mathrm{IC}_{50}=28.00 \mu \mathrm{M}\right)(P>0.05)$. The variation in glutathione content (free or mostly conjugated as trypanothione) found among $T$. cruzi strains has been also proposed as an explanation of their different susceptibility to both NX and BZ (Repetto et al. 1996), since these reduced thiols play an important role in the free radical detoxification mechanisms of trypanosomatids (Irigoin et al. 2008). Moreover, no relevant differences in glutathione content were formerly established among Tulahuen, CL and Y epimastigotes (Moncada et al. 1989) presuming a comparable response of these strains to $\mathrm{BZ}$, what 
Table 4. Trypanocidal activity of 22 and 24 on intracellular amastigotes of $\mathrm{Y}$ strain expressed as $\mathrm{IC}_{50}, \mathrm{IC}_{90}$ and SI. Compounds RP were also estimated

\begin{tabular}{llllll}
\hline \hline Compound & $\mathrm{IC}_{50}(\mu \mathrm{M})^{\mathrm{a}}$ & $\mathrm{IC}_{90}(\mu \mathrm{M})$ & $\mathrm{LC}_{50} \mathrm{CM}(\mu \mathrm{M})^{\mathrm{b}}$ & $\mathrm{SI}^{\mathrm{c}}$ & $\mathrm{RP}^{\mathrm{d}}$ \\
\hline 22 & $2 \cdot 80 \pm 0 \cdot 46$ & $6 \cdot 02 \pm 0 \cdot 30$ & $>100$ & $>35 \cdot 71$ & $1 \cdot 07$ \\
24 & $9 \cdot 02 \pm 5 \cdot 26$ & $>25$ & $>100$ & $>11 \cdot 08$ & $<0 \cdot 25$ \\
$\mathrm{BZ}$ & $1 \cdot 77 \pm 1 \cdot 11$ & $6 \cdot 46 \pm 1 \cdot 34$ & $>100$ & $>56 \cdot 50$ & - \\
\hline \hline
\end{tabular}

* Significant differences $(P<0 \cdot 05)$ compared with $\mathrm{BZ}$ (Kruskal-Wallis test).

** Significant differences $(P<0 \cdot 05)$ compared with the activity on CL-B5 (see Fonseca-Berzal et al. 2014) and Tulahuen amastigotes (Kruskal-Wallis test).

a The concentration causing 50 and $90 \%$ of $\mathrm{EI}$ inhibition $\left(\mathrm{IC}_{50}\right.$ and $\mathrm{IC}_{90}$, respectively) was estimated by plotting drug concentrations $v s \% \mathrm{EI}$. The results are expressed as the mean \pm S.D. of two independent experiments $(n=2)$.

b After $48 \mathrm{~h}$ of drug treatment.

c Selectivity indexes are defined as $\mathrm{SI}=\mathrm{LC}_{50}(48 \mathrm{~h} \mathrm{CM}$ cells $) / \mathrm{IC}_{50} \mathrm{Y}$ amastigotes.

d Relative potencies are defined as $\mathrm{RP}=\mathrm{IC}_{90} \mathrm{BZ} / \mathrm{IC}_{90}$ tested compound.
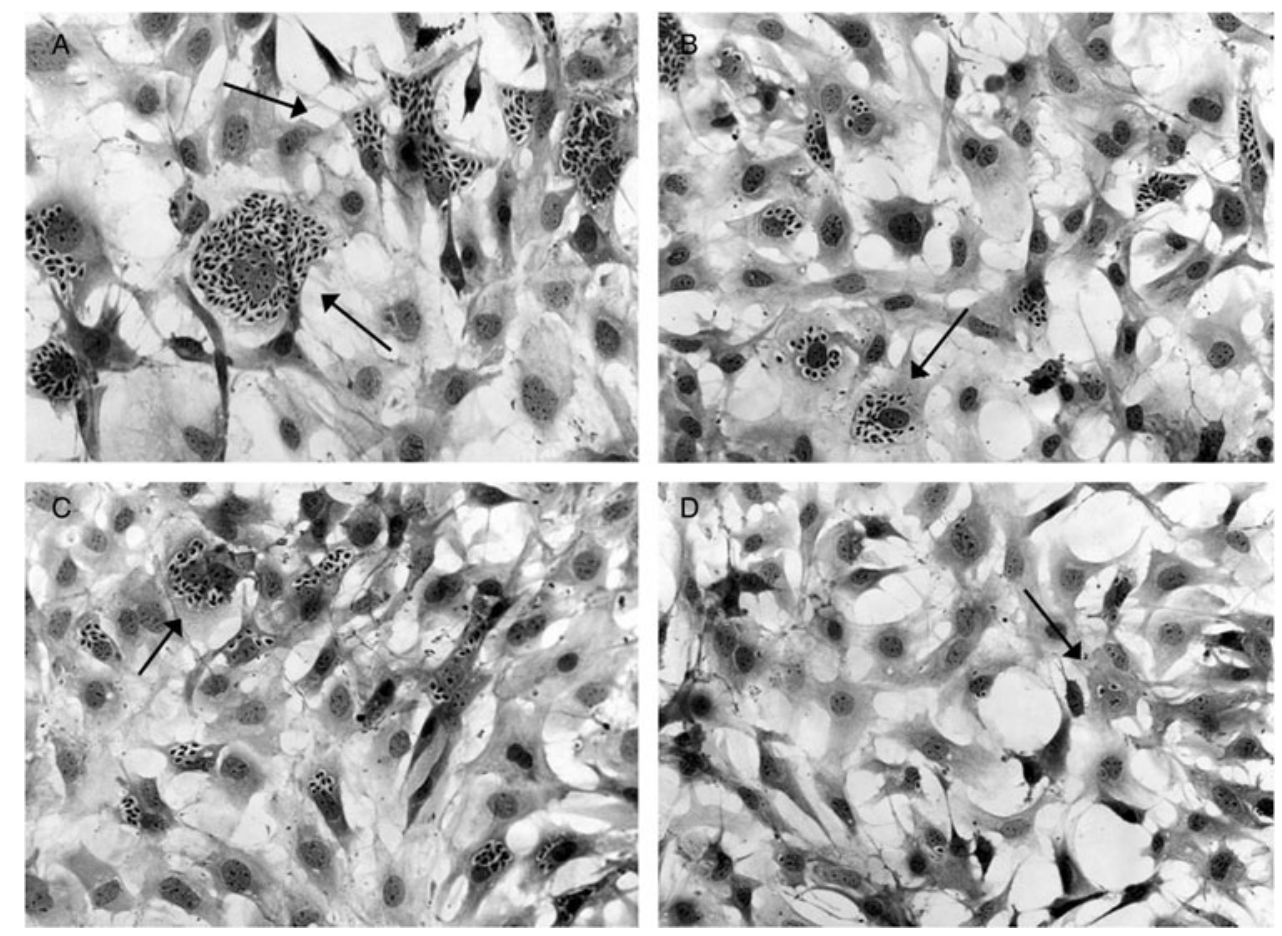

Fig. 7. Light microscopy analysis of CM infected with Y strain and treated for $48 \mathrm{~h}$ with $6 \cdot 25 \mu \mathrm{M} \mathrm{(B),} 12 \cdot 50 \mu \mathrm{M}(\mathrm{C})$ and $25 \mu \mathrm{M}$ (D) of derivative 24 , as well as untreated cells (A). The black arrows indicate intracellular amastigotes.

correlates with the similar profiles herein obtained (Table 2). Although the epimastigote is not the clinically relevant form of $T$. cruzi, the easy maintenance this model offers (Muelas et al. 2001) and the identification of an intracellular epimastigote-like form as intermediate stage within the mammalian host (Faucher et al. 1995), support the performance of pre-screening models on this stage of $T$. cruzi. Additionally, a correlation between both the activity on epimastigotes and amastigotes occurs (Vega et al. 2012; Fonseca-Berzal et al. 2014). However, compounds $\mathrm{IC}_{50}$ on these both stages usually differ in several orders of magnitude (Moreno et al. 2010). Undoubtedly, posterior inhibition assays on infective forms (i.e. trypomastigotes and amastigotes) must be carried out in vitro before advancing compounds to in vivo assays (Romanha et al. 2010), since different drug susceptibility can be registered among different stages from the same T. cruzi stock (Moraes et al. 2014).

According to these facts, we evaluated the effectiveness of derivatives 22 and 24 upon the three main forms of T. cruzi Y strain, which is routinely used in our laboratories for in vitro and in vivo drug screening models (Castillo-Garit, et al. 2012; da Silva et al. 2012; Araújo et al. 2014; FonsecaBerzal et al. 2015). The trypanocidal profile both prototypes accomplished on this strain was quite similar to that of over CL-B5 parasites (Vega et al. 2012; Fonseca-Berzal et al. 2014). However, our compounds displayed lower activity towards epimastigotes of $\mathrm{Y}$ strain $(P<0 \cdot 05)$, unlike $\mathrm{BZ}$ (Table 2). This fact may be connected with the moderately 
resistance to nitroderivative drugs attributed to T. cruzi Y (Romanha et al. 2010), contrasting with the susceptible CL Brener (Le-Senne et al. 2002). Moreover, 22 and 24 seemed to be more selective than $\mathrm{BZ}$ on extracellular epimastigotes $\left(\mathrm{SI}_{22}>\right.$ $28 \cdot 17, \mathrm{SI}_{24}>12 \cdot 62$ and $\mathrm{SI}_{\mathrm{BZ}}>3 \cdot 57$ ), since they got better effectiveness on this stage too ('Table 2).

Following the criteria proposed by DNDi (Don and Ioset, 2014), compounds that obtain $\mathrm{IC}_{50}$ values inferior than $10 \mu \mathrm{M}$ on intracellular amastigotes of TcVI or TcII are identified as putative hits for CD chemotherapy, likewise our two derivatives. These compounds formerly displayed such an outstanding activity on CL-B5 (Fonseca-Berzal et al. 2014) now confirmed in the phenotypic assay on Tulahuen $(P>0.05)$ (Table 3$)$, both TcVI strains. Besides, derivatives 22 and 24 also fulfilled it over Y amastigotes (TcII) grown in cardiac cells in vitro (Table 4 ). In fact, both compounds proved to be 10 -fold more active on the $\mathrm{Y}$ strain than toxic to mammalian cells (Don and Ioset, 2014), successfully overpassing this preliminary hit stage $\left(\mathrm{SI}_{22}>\right.$ $35 \cdot 71$ and $\mathrm{SI}_{24}>11 \cdot 08$ ).

Nevertheless, both nitroheterocycles not only displayed great activity on $T$. cruzi models, but also were considered as suitable templates for the design of novel anti-Trypanosoma brucei agents (Arán et al. 2012). However, the activity they achieved against blood trypomastigotes of both African (Arán et al. 2012) and American trypanosomes (data not shown) was lower than the obtained on the multiplying stages of T. cruzi. In the present study, our compounds achieved an improved trypanocidal profile on intracellular TcII parasites, likewise BZ (Tables 2 and 4). The activity displayed by the reference drug on $T$. cruzi $\mathrm{Y}$, could be again related to variations in thiol concentration among different stages of a unique strain (Maya et al. 1997). According to this, the higher the content of reduced thiols (i.e. epimastigotes $>$ trypomastigotes $>$ amastigotes), the less susceptible to $\mathrm{BZ}\left(\mathrm{IC}_{50}\right.$ value on epimastigotes $>$ trypomastigotes $>$ amastigotes). Actually, in a previous in vivo study we found that 22 and 24 did not entirely suppress parasitaemia through the acute disease in mice, but obtained a considerably reduction in BT levels after concluding a 5-day treatment (Fonseca-Berzal et al. 2014). The activity on BT was higher for derivative 24 either in vivo (Fonseca-Berzal et al. 2014) or in vitro models (data not shown), which conversely was the less active derivative on intracellular T. cruzi.

The results compiled in the present study corroborate these two 5-nitroindazolinone derivatives as putative antichagasic prototypes. Both compounds bore out remarkable effectiveness on the clinically relevant stage of TcII and TcVI strains with a lack of toxicity on diverse host cells that resulted in great selectivity on $T$. cruzi. Concretely, the 2-benzyl-5-nitro-1-propylindazolin-3-one (22) showed trypanocidal and cytotoxic profiles similar to those of the reference drug BZ. Further investigation directed to explore the mechanism of action triggered by these compounds is currently underway.

\section{ACKNOWLEDGEMENTS}

The authors thank the Programme for Technological Development in Tools for Health (PDTIS-Fiocruz) for the facilities. C.F.-B., J.A.E., V.J.A and A.G.-B. acknowledge M.N.C.S for kindly accepting C.F.-B. predoctoral stay in LBC/IOC/Fiocruz.

\section{FINANCIAL SUPPORT}

The present study was supported by grants from Fundação Carlos Chagas Filho de Amparo a Pesquisa do Estado do Rio de Janeiro (FAPERJ), Conselho Nacional de Desenvolvimento Científico e Tecnológico (CNPq), Fundação Oswaldo Cruz, PDTIS, PROEP/CNPq/Fiocruz, CAPES. M.N.C.S. is research fellows of CNPq and CNE researchers. This work was also supported by the UCMBSCH Research Group 'Terapia Antiparasitaria' (ref. no. 911120). Research by C.F.-B. was supported by a PICATA predoctoral fellowship and a PICATA predoctoral mobility grant of the Moncloa Campus of International Excellence (UCM-UPM \& CSIC)

\section{CONFLICT OF INTEREST}

None.

\section{ETHICAL STANDARDS}

The authors assert that all procedures contributing to this work comply with the ethical standards of the relevant national and institutional guides on the care and use of laboratory animals.

\section{REFERENCES}

Aguilera-Venegas, B., Olea-Azar, C., Arán, V. J. and Speisky, H. (2013). Indazoles: a new top seed structure in the search of efficient drugs against Trypanosoma cruzi. Future Medicinal Chemistry 5, 1843-1859.

Arán, V. J., Ochoa, C., Boiani, L., Buccino, P., Cerecetto, H., Gerpe, A., González, M., Montero, D., Nogal, J. J., GómezBarrio, A., Azqueta, A., López de Ceráin, A., Piro, O. E. and Castellano, E. E. (2005). Synthesis and biological properties of new 5nitroindazole derivatives. Bioorganic and Medicinal Chemistry 13, $3197-$ 3207.

Arán, V. J., Kaiser, M. and Dardonville, C. (2012). Discovery of nitroheterocycles active against African trypanosomes. In vitro screening and preliminary SAR studies. Bioorganic and Medicinal Chemistry Letters 22 , 4506-4516.

Araújo, J. S., da Silva, C. F., Batista, D. G., da Silva, P. B., Batista, M. M., Aiub, C. A. F., da Silva, M. F., Araújo-Lima, C. F., Banerjee, M., Farahat, A. A., Stephens, C. E., Kumar, A., Boykin, D. W. and Soeiro, M. N. C. (2014). In vitro and in vivo studies of the biological activity of novel arylimidamides against Trypanosoma cruzi. Antimicrobial Agents and Chemotherapy 58, 4191-4195.

Boiani, M., Boiani, L., Denicola, A., Torres de Ortiz, S., Serna, E. Vera de Bilbao, N., Sanabria, L., Yaluff, G., Nakayama, H., Rojas de Arias, A., Vega, C., Rolón, M., Gómez-Barrio, A., Cerecetto, H. and González, M. (2006). 2H-Benzimidazole 1,3-dioxide derivatives: a new family of water-soluble anti-trypanosomatid agents. Fournal of Medicinal Chemistry 49, 3215-3224.

Boiani, L., Gerpe, A., Arán, V. J., Torres de Ortiz, S., Serna, E., Vera de Bilbao, N., Sanabria, L., Yaluff, G., Nakayama, H., Rojas de 
Arias, A., Maya, J. D., Morello, J. A., Cerecetto, H. and González, M. (2009). In vitro and in vivo antitrypanosomatid activity of 5-nitroindazoles. European Fournal of Medicinal Chemistry 44, 1034-1040.

Buckner, F. S., Verlinde, C. L. M. J., La Flamme, A. C. and Van Voorhis, W. C. (1996). Efficient technique for screening drugs for activity against Trypanosoma cruzi using parasites expressing $\beta$-galactosidase Antimicrobial Agents and Chemotherapy 40, 2592-2597.

Castillo-Garit, J. A., del Toro-Cortés, O., Kouznetsov, V. V., Puentes, C. O., Romero Bohórquez, A. R., Vega, M. C., Rolón, M., Escario, J. A., Gómez-Barrio, A., Marrero-Ponce, Y., Torrens, F. and Abad, C. (2012). Identification in silico and in vitro of novel trypanosomicidal drug-like compounds. Chemical Biology and Drug Design 80, $38-45$.

Castro, J. A., Montalto de Mecca, M. and Bartel, L. C. (2006). Toxic side effects of drugs used to treat Chagas' disease (American trypanosomiasis). Human and Experimental Toxicology 25, 471-479.

da Silva, C. F., Batista, D. G., Oliveira, G. M., de Souza, E. M., Hammer, E. R., da Silva, P. B., Daliry, A., Araújo, J. S., Britto, C., Rodrigues, A. C., Liu, Z., Farahat, A. A., Kumar, A., Boykin, D. W. and Soeiro, M. N. C. (2012). In vitro and in vivo investigation of the efficacy of acrylimidamide DB1831 and its mesylated salt form -DB1965against Trypanosoma cruzi infection. PLoS ONE 7, e30356.

da Silva, C. F., Batista, M. M., Mota, R. A., de Souza, E. M., Stephens, C. E., Som, P., Boykin, D. W. and Soeiro, M. N. C. (2007) Activity of 'reversed' diamidines against Trypanosoma cruzi 'in vitro'. Biochemical Pharmacology 73, 1939-1946.

Don, R. and Ioset, J. R. (2014). Screening strategies to identify new chemical diversity for drug development to treat kinetoplastid infections. Parasitology 141, 140-146.

Duran-Rehbein, G. A., Vargas-Zambrano, J. C., Cuéllar, A., Puerta, C. J. and González, J. M. (2014). Mammalian cellular culture models of Trypanosoma cruzi infection: a review of the published literature. Parasite 21, 38

Emter, R. and Natsch, A. (2015). A fast Resazurin-based live viability assay is equivalent to the MTT-test in the KeratinoSens assay. Toxicology In Vvitro 29, 688-693.

Faucher, J. F., Baltz, T. and Petry, K. G. (1995). Detection of an 'epimastigote-like' intracellular stage of Trypanosoma cruzi. Parasitology Research 81, 441-443.

Folch-Cano, C., Olea-Azar, C., Arán, V. J. and Díaz-Urrutia, C. (2010) ESR and electrochemical study of 1,2-disubstituted 5-nitroindazolin-3-one and 2-substituted 3 -alkoxy-5-nitro- $2 \mathrm{H}$-indazoles: reactivity and free radical production capacity in the presence of biological systems. Spectrochimica Acta Part A: Molecular and Biomolecular Spectroscopy 75, 375-380.

Fonseca-Berzal, C., Escario, J. A., Arán, V. J. and Gómez-Barrio, A (2014). Further insights into biological evaluation of new anti-Trypanosoma cruzi 5-nitroindazoles. Parasitology Research 113, 1049-1056.

Fonseca-Berzal, C., Palmeiro-Roldán, R., Escario, J. A., Torrado, S. Arán, V. J., Torrado-Santiago, S. and Gómez-Barrio, A. (2015). Nove solid dispersions of benznidazole: preparation, dissolution profile and biological evaluation as alternative antichagasic drug delivery system. Experimental Parasitology 149, 84-91.

Irigoin, F., Cibils, L., Comini, M. A., Wilkinson, S. R., Flohe, L. and Radi, R. (2008). Insights into the redox biology of Trypanosoma cruzi Trypanothione metabolism and oxidant detoxification. Free Radical Biology and Medicine 45, 733-742.

Le-Senne, A., Muelas-Serrano, S., Fernández-Portillo, C. Escario, J. A. and Gómez-Barrio, A. (2002). Biological characterization of a $\beta$-galactosidase expressing clone of Trypanosoma cruzi CL strain. Memórias do Instituto Oswaldo Cruz 97, 1101-1105.

Maya, J. D., Repetto, Y., Agosin, M., Ojeda, J. M., Téllez, R., Gaule, C. and Morello, A. (1997). Effects of nifurtimox and benznidazole upon glutathione and trypanothione content in epimastigote, trypomastigote and amastigote forms of Trypanosoma cruzi. Molecular and Biochemical Parasitology 86, 101-106.

Meirelles, M. N., de Araújo-Jorge, T. C., Miranda, C. F., de Souza, W. and Barbosa, H. S. (1986). Interaction of Trypanosoma cruzi with heart muscle cells: ultrastructural and cytochemical analysis of endocytic vacuole formation and effect upon myogenesis in vitro. European Fournal of Cell Biology 41, 198-206.

Moncada, C., Repetto, Y., Aldunate, J., Letelier, M. E. and Morello, A. (1989). Role of glutathione in the susceptibility of Trypanosoma cruzi to drugs. Comparative Biochemistry and Physiology. Part C: Comparative Pharmacology 94, 87-91.
Moraes, C. B., Giardini, M. A., Kim, H., Franco, C. H., AraujoJunior, A. M., Schenkman, S., Chatelain, E. and Freitas-Junior, L. H. (2014). Nitroheterocyclic compounds are more efficacious than CYP51 inhibitors against Trypanosoma cruzi: implications for Chagas disease drug discovery and development. Scientific Reports 4, 4703

Moreno, M., D'ávila, D. A., Silva, M. N., Galvão, L. M., Macedo, A. M., Chiari, E., Gontijo, E. D. and Zingales, B. (2010). Trypanosoma cruzi benznidazole susceptibility in vitro does not predict the therapeutic outcome of human Chagas disease. Memórias do Instituto Oswaldo Cruz 105, 918-924

Muelas, S., Di Maio, R., Cerecetto, H., Seoane, G., Ochoa, C., Escario, J. A. and Gómez-Barrio, A. (2001). New thiadiazine derivatives with activity against Trypanosoma cruzi amastigotes. Folia Parasitologica 48, 105-108.

Muro, B., Reviriego, F., Navarro, P., Marín, C., Ramírez-Macías, I., Rosales, M. J., Sánchez-Moreno, M. and Arán, V. J. (2014). New perspectives on the synthesis and antichagasic activity of 3-alkoxy1-alkyl-5-nitroindazoles. European Fournal of Medicinal Chemistry 74, $124-134$

Repetto, Y., Opazo, E., Maya, J. D., Agosin, M. and Morello, A. (1996). Glutathione and trypanothione in several strains of Trypanosoma cruzi: effect of drugs. Comparative Biochemistry and Physiology. Part B Biochemistry and Molecular Biology 115, 281-285

Rolón, M., Vega, C., Escario, J. A. and Gómez-Barrio, A. (2006) Development of resazurin microtiter assay for drug sensibility testing of Trypanosoma cruzi epimastigotes. Parasitology Research 99, 103-107.

Romanha, A. J., Castro, S. L., Soeiro, M. N. C., Lannes-Vieira, J., Ribeiro, I., Talvani, A., Bourdin, B., Blum, B., Olivieri, B., Zani, C., Spadafora, C., Chiari, E., Chatelain, E., Chaves, G., Calzada, J. E., Bustamante, J. M., Freitas-Junior, L. H., Romero, L. I., Bahia, M. T., Lotrowska, M., Soares, M., Andrade, S. G., Armstrong, T., Degrave, W. and Andrade, Z. A. (2010). In vitro and in vivo experimental models for drug screening and development for Chagas disease. Memórias do Instituto Oswaldo Cruz 105, 233-238.

Schmunis, G. A. and Yadon, Z. E. (2010). Chagas disease: a Latin American health problem becoming a world health problem. Acta Tropica 115, 14-21.

Silva, L. H. and Nussenzweig, V. (1953). Sobre uma cepa de Trypanosoma cruzi altamente virulenta para o camundongo branco. Folha Clinica e Biologica 20, 191-207.

Soeiro, M. N. C., de Souza, E. M., da Silva, C. F., Batista, D. G., Batista, M. M., Pavão, B. P., Araújo, J. S., Aiub, C. A. F., da Silva, P. B., Lionel, J., Britto, C., Kim, K., Sulikowski, G. and Hargrove, T. Y. (2013). In vitro and in vivo studies of the antiparasitic activity of sterol $14 \alpha$-demethylase (CYP51) inhibitor VNI against drugresistant strains of Trypanosoma cruzi. Antimicrobial Agents and Chemotherapy 57, 4151-4163.

Teixeira, A. R., Nascimento, R. J. and Sturm, N. R. (2006). Evolution and pathology in Chagas disease - a review. Memórias do Instituto Oswaldo Cruz 101, 463-491

Urbina, J. A. (2015). Recent clinical trials for the etiological treatment of chronic Chagas disease: advances, challenges and perspectives. Fournal of Eukaryotic Microbiology 62, 149-156.

Vega, M. C., Rolón, M., Montero-Torres, A., Fonseca-Berzal, C., Escario, J. A., Gómez-Barrio, A., Gálvez, J., Marrero-Ponce, Y. and Arán, V. J. (2012). Synthesis, biological evaluation and chemometric analysis of indazole derivatives. 1,2-disubstituted 5-nitroindazolinones, new prototypes of antichagasic drug. European Fournal of Medicinal Chemistry 58, 214-227.

World Health Organization (2015). Investing to Overcome the Global Impact of Neglected Tropical Diseases, Third WHO Report on Neglected Tropical Diseases. Department of Control of Neglected Tropical Diseases, World Health Organization, Geneva.

Xu, M., McCanna, D. J. and Sivak, J. G. (2015). Use of the viability reagent PrestoBlue in comparison with alamarBlue and MTT to assess the viability of human corneal epithelial cells. Fournal of Pharmacological and Toxicological Methods 71, 1-7.

Zingales, B., Miles, M. A., Moraes, C. B., Luquetti, A., Guhl, F., Schijman, A. G. and Ribeiro, I. (2014). Drug discovery for Chagas disease should consider Trypanosoma cruzi strain diversity. Memórias do Instituto Oswaldo Cruz 109, 828-833.

Zwolak, I. (2014). Comparison of three different cell viability assays for evaluation of vanadyl sulphate cytotoxicity in a Chinese hamster ovary K1 cell line. Toxicology and Industrial Health. doi:10.1177/0748233714544190. 